\title{
High-Frequency Neuronavigated Repetitive Transcranial Magnetic Stimulation for Depression in Major Neurocognitive Disorders: A Randomized Controlled Trial
}

Hanna Lu

Chinese University of Hong Kong https://orcid.org/0000-0002-9090-258X

Cuichan Lin

The Chinese University of Hong Kong

\section{Sandra Sau Man Chan}

The Chinese University of Hong Kong

Suk Ling Ma

The Chinese University of Hong Kong

Vincent Chung Tong Mok

The Chinese University of Hong Kong

Lin Shi

The Chinese University of Hong Kong

Defeng Wang

The Chinese University of Hong Kong

Arthur Dun-Ping Mak

The Chinese University of Hong Kong

Linda Chiu Wa Lam ( $\Delta$ cwlam@cuhk.edu.hk)

The Chinese University of Hong Kong

\section{Research}

Keywords: Neurocognitive disorder, depression, rTMS, neuronavigation, randomized clinical trial

Posted Date: October 19th, 2020

DOl: https://doi.org/10.21203/rs.3.rs-91498/v1

License: (1) This work is licensed under a Creative Commons Attribution 4.0 International License. Read Full License 


\section{Abstract}

Background: We evaluated the safety and efficacy of high-frequency repetitive transcranial magnetic stimulation (HF-rTMS) in major neurocognitive disorder (NCD) patients with depression.

Methods: In this single blind, sham-controlled clinical trial, 58 patients of major NCD with clinically significant depressive symptoms (Cornell Depression Scale for Dementia, CSDD $\geq 7$ ) were enrolled and randomly (1:1) assigned for a three-week treatment of either active rTMS $(10 \mathrm{~Hz}, 120 \%$ motor threshold, 1500 pulses/session) or sham (placebo-controlled) rTMS treatment. Structural magnetic resonance imaging (MRI) scans were employed to localize the stimulation targets and guide the TMS coil during the treatment. Severity of depressive symptoms, global cognitive function and the serum brain-derived neurotrophic factor (BDNF) level were evaluated at baseline, post-intervention and 6-weeks and 12-weeks follow-ups.

Results: Twenty-seven participants were randomized to receive active rTMS and 28 to receive sham rTMS. Both groups showed improvement in depressive symptoms and cognitive function after 3 weeks rTMS treatment. Logistic regression analysis revealed that older age $(p=0.016)$, higher baseline cognitive function $(p=0.041)$ and randomization group $(p=0.025)$ were associated with the remission of depression (Nagelkerke $R^{2}=0.42$ ).

Conclusions: A 3-week course of $10 \mathrm{~Hz}$ rTMS appears to be a safe intervention for bringing quicker remission of depression for neurocognitive disorder patients.

Trial registration: ClinicalTrials.gov, ChiCTR-IOR-16008191. Registered on March 30, 2016.

\section{Background}

The burden of dementia is complicated by the presence of comorbid neuropsychiatric symptoms, of which depression is one of the prominent and distressing symptoms [1, 2]. Meta-analyses estimated that depression affects nearly $25 \%$ of mild neurocognitive disorder (NCD) patients, and $40 \%$ in clinical populations $[3,4]$. Another study focusing on community-residing senior adults reported that over $60 \%$ of seniors in Hong Kong suffering from the co-existence of NCD and depression [5]. It is worth noting that depressive symptoms occur through the disease course from prodromal to severe phase of dementia [6].

The aetiology for depressive disorder is complex. Depression in NCD is further complicated by underlying neurodegeneration, inflammatory and immune reactions associated with the specific dementia pathologies [7]. Effective management of depressive symptoms in people with NCD requires more than functional restoration of neurotransmitter imbalance in primary depressive disorders. It requires an adaptive process with degenerating central nervous system, of which the impaired neuroplasticity is considered as the overlapping neurobiological mechanism that underlies cognitive impairments and depression [7]. Moreover, it has been proposed that the progression of cognitive impairment is related to the dysfunction of frontal cortex, which may account for the aetiology of depression in different subtypes 
of NCD $[8,9]$. Magnetic resonance imaging (MRI) studies also reported the presence hypoperfusion and disturbed connectivity of left dorsolateral prefrontal cortex (DLPFC) in NCD comorbid depression [10-12].

Transcranial magnetic stimulation (TMS) is a non-invasive brain stimulation technique that has generated considerable attention as a safe and effective non-pharmacological treatment of several neuropsychiatric and neurological disorders, including major depressive disorder [13], Alzheimer's disease [14], vascular disease [15], The putative mechanism on the therapeutic effect of TMS is related to the modulation of neuroplasticity by inducing the flow of electric current at the surface of cerebral cortex through sequence of powerful, rapidly changing magnetic field pulses [16]. There is preliminary evidence to support the antidepressant effect of high-frequency repetitive TMS (HF-rTMS) over left DLPFC for individuals with vascular depression not responsive to pharmacological treatment $[17,18]$. On the cellular level, significant changes of synaptic plasticity with the presence of increased expression of serum brainderived neurotrophic factor (BDNF) after successive rTMS treatment in mouse models of brain ageing and dementia [19]. Interestingly, several studies using TMS to enhance cognitive function in dementia patients, also found an improvement in depressive symptoms [20,21]. Meanwhile, it should be noted that individual differences in the treatment of depression inferred from observational studies and clinical trials could present considerable problems in interpreting the effect of TMS, such as placebo effect [22], neuroanatomical heterogeneity due to age-related brain atrophy [23].

Meanwhile, it remains unclear about the efficacy and sustainability of placebo-controlled HF-rTMS in NCD patients suffering from depression. Thus, the aims of this study included: (1) to evaluate the feasibility, safety and efficacy of neuronavigated 3-week HF-rTMS in the treatment of depression in major NCD patients; (2) to examine the potential predictors of the sustainable effect of HF-rTMS on depressive symptoms and cognitive function during a 2-month follow-up period; (3) to investigate the paralleled changes in serum BDNF level after HF-rTMS treatment.

\section{Methods}

\section{Study design}

This study was a single-blind (participant), sham-controlled randomized clinical trial conducted at the Chen Wai Wai Vivien Foundation Therapeutic Physical Mental Exercise Centre at the Department of Psychiatry of the Chinese University of Hong Kong from August 2016 to March 2019 (ChiCTR-IOR16008191). The logistics of this trial was followed the Consolidated Standards of Reporting Trials (CONSORT) flow diagram (Figure 1). Eligible participants were enrolled and randomly assigned to a threeweek intervention of either active rTMS or sham rTMS. After the intervention, participants were followed for 8 weeks.

The inclusion criteria include [24]: (1) Aged from 60 to 90 years old; (2) DSM-5 diagnosis of either major neurocognitive disorder due to Alzheimer's disease (NCD-AD) or major neurocognitive disorder due to vascular disease (NCD-vascular), including significant cognitive decline in the core domains of cognitive function (attention, memory, perceptual-motor, language and executive function), and with clinical or 
neuroimaging features indicative of either $A D$ or small vessel disease; (4) Cornell scale for depression in dementia (CSDD) with a rating score of 7 or above, indicating clinically depression [25]. Throughout the trial, anti-dementia and other psychotropic medication were kept constant and recorded.

We excluded the participants who had a history of primary bipolar or other psychotic disorders, and major neurological disorders, including stroke, transient ischemic attack or traumatic brain injury. The participants were also excluded if they were unable to attend the MRI scanning and rTMS session due to contraindications (e.g., metal on or inside the body).

\section{Randomization and masking}

We randomly assigned the participants (1:1) to receive either active rTMS or sham rTMS. We masked the participants, caregivers, and assessors at the center to intervention assignment. The research staff performing the rTMS was open to the randomization status. The participants who voluntarily withdrew or discontinued the rTMS treatment due to a serious adverse event, no further data from the point of withdrawal was collected.

\section{Procedures}

\section{Pre-intervention and preparation}

Structural magnetic resonance imaging (sMRI) data was acquired using a 3T MRI scanner (Philips Healthcare, Best, Netherlands) at the Prince of Wales Hospital in Hong Kong. The T1-weighted magnetization prepared rapid gradient echo (MPRAGE) sequence was empirically optimized for the greywhite contrast, with repetition time $(T R)=7.6 \mathrm{~ms}$, echo time $(T E)=3.5 \mathrm{~ms}$, field of view $(F O V)=230 \mathrm{~mm}$, thickness $=0.6 \mathrm{~mm}$, axial acquisition with a $256 \times 256 \times 192$ matrix, flip angle $(\mathrm{FA})=15^{\circ}$ and the voxel size of $1 \mathrm{~mm} \times 1 \mathrm{~mm} \times 1 \mathrm{~mm}$.

\section{Neuronavigated rTMS intervention}

As shown in Figure 2, the pipeline of neuronavigated rTMS intervention contained three parts: (1) Reconstruction and localization: Based on individual SMRI data, we firstly reconstructed the scalp and cortex, and then localized the targets of interest labelled with the Montreal Neurological Institute (MNI) coordinates $(x, y, z)$ (Figure 2A); (2) Measurement of motor threshold (MT) [26]: MT was measured through the single-pulse TMS over left primary motor cortex (M1, hand area) prior to the first treatment. The MT was defined as the minimum stimulus intensity required to evoke an motor evoked potential (MEP) with a peak-to-peak amplitude $\geq 50 \mu \mathrm{V}$ in at least 5 out of 10 consecutive trials recorded in the electromyography (EMG) system (Figure 2B); (3) Treatment: $10 \mathrm{~Hz}$ high-frequency rTMS over left DLPFC was provided by using Magstim Super Rapid stimulator (Magstim, UK) that generates short duration biphasic pulses. The Magstim 70 mm figure-8 coil (AirFilm ${ }^{\circledR}$ Coil AFC) was held in place with a custommade stand tangential to the scalp with the handle pointing back and away from the midline at $45^{\circ}$ (Figure 2C). 
An MRI-to-head coregistration was performed to localize the anatomical landmarks individually. Once successfully coregistered, a real-time infrared tracking system (Polaris Northern Digital) was used to monitor the coil position and the orientation of the stimulation targets. As mentioned, we targeted the location of left DLPFC with the MNI coordinates as $[x=-46, y=45, z=38]$, being carefully to locate this region within the grey matter on the top of middle frontal gyrus in the Brainsight system [26-28].

The HF-rTMS intensity was set at $120 \%$ of MT, and 30 trains of $10 \mathrm{~Hz}$ stimulation with 5 -second stimulation duration and 25-second interval. This protocol resulted in the delivery of 1500 pulses in each rTMS treatment session. The treatment schedule was 1 session per day, 5 days per week, lasting for 3 weeks. In the condition of sham rTMS, same coil was used for single blind trial in order to compensate for the acoustic and other non-specific effect [29].

\section{Intervention schedule}

Fifteen sessions of HF-rTMS were conducted by trained clinician. Outcome measures were collected at T0 (baseline), T1 ( $3^{\text {rd }}$ week, post-intervention), T2 ( $6^{\text {th }}$ week, 3 weeks post-intervention, $)$ and T3 (1 ${ }^{\text {th }}$ week, 6 weeks post-intervention). All research staffs were trained to ensure the data accuracy, consistency and completeness.

\section{Outcome measures}

\section{Primary outcomes}

1. Severity of depression: Cornell Scale for Depression in Dementia (CSDD) was used for assessing depressive symptoms. In this study, a cutoff score of 7 indicated clinically significant depression in NCD patients. Responders were defined as having a $50 \%$ reduction in the CSDD scores from the baseline [30,31].

2. Global cognitive function: The Montreal Cognitive Assessment (MoCA) Hong Kong version was used to measure the global cognitive changes associated with intervention [32,33].

\section{Secondary outcomes:}

1. Neuropsychiatric symptoms: The Chinese Neuropsychiatric Inventory (NPI) was used to assess the changes of neuropsychiatric symptoms across twelve domains [34].

2. Quality of life: Quality of Life in Alzheimer's disease scale (QoL-AD), including both patient and caregiver components, is a 13-item scale designed for generic measure of the quality of life in dementia patients [35].

3. Brain-derived neurotrophic factor (BDNF) level was used as a peripheral marker of neuroplastic response [36]. Serum BDNF was assayed in duplicate according to the manufacturer's instructions (R\&D Systems, Inc., Minneapolis, MN, USA).

4. Adverse events: Adverse Event Checklist (AEC) covering the potential adverse effect associated with HF-rTMS administration was conducted in each assessment point through the study [37]. 


\section{Statistical analysis}

Analyses were on an intention-to-treat basis. Linear mixed models were conducted to assess the differences between intervention groups on the primary and secondary outcomes at each time point. Treatment, time points, and their interaction were modeled as fixed effect. Participants were modeled as random effect at different time points. Score changes of depression score and global cognition from baseline to each follow-up point and intervention group were tested with occasions (time points) at level one and participants at level two. Cohen's $d$ was calculated as a measure of effect size, of which Cohen's $d \geq 0.8$ refers to a strong effect, $0.5 \leq$ Cohen's $d<0.8$ refers to a moderate effect and Cohen's $d<0.5$ means the effect is statistically significant but weak [38]. All analyses were performed using SPSS Statistics 24.0 (IBM, Armonk, NY).

\section{Results}

\section{Demographics and clinical characteristics}

Between June 2016 and March 2019, 100 participants were screened for the eligibility, of which 55 were randomly assigned to receive active rTMS $(n=27)$ or sham rTMS $(n=28)$ (Figure 1$)$. During the whole study period, 2 cases (7.4\%) of 27 participants in the active rTMS group and 1 (3.6\%) of 28 in the sham rTMS group withdrew. Therefore, 52 participants were included in the efficacy analysis.

Baseline demographics and clinical characteristics of participants were balanced between the randomized groups (Table 1). The mean age of sham rTMS group (73.7 \pm 7.2 years) was older than the active rTMS group ( $69.2 \pm 7.3$ years) ( $t$-test, $p=0.02$ ). There were no group differences in sex, years of education, severity of depression and global cognitive function at baseline. Reviews of regular medication showed no differences of antipsychotic, anti-hypertension, antidepressant medications taken between active and sham rTMS groups.

\section{Outcome measures}

For the clinical outcome, there were significant reductions of depressive symptoms (measured by CSDD) and improvements in global cognitive function (measured by MoCA) in both intervention groups. The score changes of CSDD from baseline to T1 was 3.24 (SE 0.71) for active rTMS group (Cohen's $d=0.92$; $p<0.001$ ) and 2.15 (SE 0.62) for sham rTMS group (Cohen's $d=0.66 ; p=0.002$ ). The score change of MoCA from baseline to T1 was 1.96 (SE 0.42) for active rTMS group (Cohen's $d=0.93 ; p<0.001$ ) and 2.07 (SE 0.61) for sham rTMS group (Cohen's $d=0.65 ; p=0.002$ ). The improvements of mood and global cognitive function persisted in 12 weeks (T3) in both groups (active rTMS: CSDD: Cohen's $d=0.74 ; p<$ 0.001; MoCA: Cohen's $d=0.5 ; p<0.001)$, but the magnitude of improvement declined over time, particularly in sham rTMS group (sham rTMS: CSDD: Cohen's $d=0.49 ; p=0.028$; MoCA: Cohen's $d=0.26$; $p=0.012$ ). 
There was no group difference in the score changes of CSDD at 3 weeks in active and sham rTMS group (Cohen's $d=0.33 ; p=0.249$ ) (Figure 3A). Similarly, no group differences were found in the score changes of MoCA (Cohen's $d=0.04 ; p=0.878$; Figure 3B). Additionally, score changes of CSDD and MoCA at either 6 weeks or 12 weeks were not different between the groups (Cohen's $d=0.31 ; p=0.279$ ).

Across all the follow-up time points ( $\mathrm{T} 1, \mathrm{~T} 2$ and $\mathrm{T} 3)$, there were no group differences in the primary outcomes of the raw scores of CSDD and MoCA (Table 3). No significant differences were observed for the scores of NPI, QoL-AD and adverse events between the groups.

\section{Response rate and remission rate}

Participants in active rTMS group had a $25.5 \%$ reduction in their CSDD score at 12 weeks compared with $21.8 \%$ reduction in sham rTMS group. Response rate, defined as $\geq 50 \%$ improvement on CSDD score, was observed in 4 cases (16\%) for active rTMS group versus 4 (14\%) for sham rTMS group at 3 weeks and 12 weeks (Figure 4A).

The proportion of participants achieving clinical remission was evaluated. At 3 weeks, 8 cases (32\%) of the active rTMS group and 3 cases $(11 \%)$ of the sham rTMS group were classified as in remission $(p=$ 0.07). At the endpoint of this study (T3), 5 cases (20\%) of the active rTMS group and 5 cases (18\%) of the sham rTMS group were classified as in remission (Figure 4B). The proportion of cases with remission did not differ between two groups at 6 weeks and 12 weeks. Logistic regression analysis revealed that older age $(p=0.016)$, higher baseline cognitive function (MoCA, $p=0.041)$ and randomization group (i.e., active rTMS) $(p=0.025)$ were associated with clinical remission after the intervention at 3 weeks (Nagelkerke $R^{2}=0.42$ ). Randomization group was not a significant factor for clinical remission at 6 weeks and 12 weeks.

\section{BDNF levels}

There were no associations between baseline BDNF level and depression or cognitive scores. At T1, the BDNF level of active rTMS group showed a marginally significant increase compared with sham rTMS group $(p=0.05)$. There was no group difference in the BDNF levels across all the time points (T0 to T3). Using age and education as covariates, a weak but statistically non-significant association between BDNF level and decreased CSDD score was observed in active rTMS group $(r=-0.677, p=0.065)$ at T1, but not detected in sham rTMS group $(r=-0.537, p=0.136)$.

\section{Discussion}

The main purpose of this study was to examine the feasibility, safety and efficacy of MRI imaged-guided high-frequency rTMS over left DLPFC for treating depressive symptoms in neurocognitive disorders. We found that a 3-week 15 session HF-rTMS treatment, either active HF-rTMS or sham HF-rTMS, demonstrated a positive effect on ameliorating depressive symptoms and enhancing the global cognitive 
function in major NCD patients with depression, but active rTMS group appeared to have a higher remission rate than sham rTMS group after 3 weeks of intervention.

The antidepressant effect of HF-rTMS has been demonstrated in the treatment of major depression in young and middle-age adults, and there is also accelerating evidence to support its use in late-life depression and depression in neurologic disorders [39-41]. Although a few investigations found that there were reductions of depressive symptoms in older adults with treatment-resistant depression or vascular depression after rTMS treatment [17, 42], the clinical effectiveness of rTMS in these studies were not consistent. For instance, Figiel et al conducted an open-label trial and found that HF-rTMS can produce a modest antidepressant effect in older patients with a response rate of $23 \%$, which was lower than the patients with younger age (56\%) [43]. Later, Fabre et al reported a similar result but with a higher response rate (45\%) in elderly patients [44]. With respect to placebo-controlled trials, Mosimann et al found that the efficacy of active rTMS was not superior to sham rTMS for treating depression in older adults [45], and similarly, Manes et al reported no reductions in depression severity after active rTMS treatment in elderly patients [46].

Stemming from the heterogeneous results across the rTMS studies, some conclusions for rTMS treatment could be outlined here: (1) the effectiveness of sham rTMS and (2) age-related rTMS effect on depressive symptoms. Of the reported cases, the differences of response rate may be related to the absence of sham condition; whereas the available randomized controlled trials (RCTs) indicate that active rTMS has only mild to modest improvements on depression and a comparable effect size in comparison with sham rTMS. Our results confirmed the similar response rate in active rTMS group (18\%) and sham rTMS group (16\%) at 3 weeks and 12 weeks. The remission rate in active rTMS group (32\%) was higher than sham rTMS group (11\%) at 3 weeks. Although the antidepressant effect in both intervention groups was gradually diminished over time, our findings suggest that randomization group is a key factor related to the positive clinical outcome in major NCD with depression. Meanwhile, sham intervention is an important consideration for RCTs as placebo effect should not be ignored. Especially, recent study has reported that sham rTMS can change the underlying brain connectivity just as active rTMS did when treating depression [47].

In terms of age-related rTMS effect, the magnitude of the antidepressant effect of rTMS varies considerably in depressed patients across the age spectrum [17,39-45]. The question as to whether age can be used as a predictor to the efficacy of rTMS is one of the topics fiercely debated in recent years. On one hand, initial RCTs indicate that old age may be a poor predictor of the response to rTMS, with lower response rate in elderly patients $[45,46]$, of which this conclusion is not supported by meta-analyses and recent report [48-50]. On the other hand, advanced age has been shown to correlate with better response to rTMS $[50,51]$. We found that the patients with older age appeared to have a better response to rTMS at 3 weeks. Notably, the link between age and treatment-induced effect in depressed patients has also been highlighted by Cuijpers and colleagues [52]. This meta-analysis found that cognitive behavioral therapy was less effective in children and adolescents rather than adults. Consequently, it seems that age, as a 
predictive factor, is not just evident in, but also specific to, treatment-induced effect, which is worth to investigate in future studies.

Upon the antidepressant effect of HF-rTMS, another interesting finding is a synergistic effect on cognitive function across all the follow-up observations. In fact, it is not surprising to observe the amelioration of depression, is often in association with enhanced cognitive function [44], because of the overlapping mechanisms of depression and cognitive deficits, such decreased cortical activities [10,11], impaired neuroplasticity [12]. Accordingly, it is quite reasonable to observe the changes of mood and cognitive function after active rTMS, as the mechanisms underlying the treatment effect of HF-rTMS are associated with an enhanced cortical excitability and neuroplasticity [25]. Interestingly, the synergistic effect on global cognitive function and depression was also found in sham rTMS group.

rTMS, as an adjuvant treatment, that offers additional benefits on cognitive function are worth considering, especially in the context of neurodegeneration. Even though this phenomenon has been reported by a few small-scale studies [14,53], the dissociable cognitive trajectories in active and sham rTMS groups indicate that rTMS may have differential effect on mood regulation and cognitive function characterizing with neuroplasticity. Unfortunately, although the serum BDNF level of active rTMS group showed a marginally significant increase compared with sham rTMS group, no noticeable associations were detected between baseline BDNF level and depression or cognitive scores.

This study has limitations. The absence of the subtypes of major NCD patients may limit the interpretations of the treatment efficacy of rTMS in disease-specific populations. Another important limitation was a confounder of routine pharmacological treatments during the study period. Although we did not detect any differences in the medications administered to the participants of two randomized groups, there might have potential impact on the efficacy of HF-rTMS. On the other hand, this study has strengths including personalized neuronavigation, sham condition and real-time feedbacks of coil position during the rTMS. These factors led to an efficient and precise study design that we hope will address the aims.

\section{Conclusions}

A three-week $10 \mathrm{~Hz}$ rTMS over left DLPFC, either active or sham, resulted in reduction of depressive symptoms and enhancement of global cognitive function in elderly comorbid major neurocognitive disorder and depression. The patients received active rTMS showed higher remission rate than the ones received sham rTMS. The findings give support for high-frequency rTMS to be considered as an adjuvant treatment for depressive symptoms in cognitively compromised elderly patients, especially for its rapid antidepressant effect bridging the therapeutic gap when antidepressant takes two weeks or longer to take effect on mood, or through the choices of antidepressant when adverse effect occur.

\section{Abbreviations}


$A D$ : Alzheimer's disease

ADL: Activities of daily living

AEC: Adverse event checklist

BDNF: Brain-derived neurotrophic factor

CSDD: Cornell scale for depression in dementia

DLPFC: Dorsolateral prefrontal cortex

EMG: Electromyography

MNI: Montreal neurology institute

MoCA: Montreal cognitive assessment

MRI: Magnetic resonance imaging

MT: Motor threshold NCD: Neurocognitive disorder

NPI: Neuropsychiatric inventory

MEP: Motor evoked potential

QoL-AD: Quality of Life in Alzheimer's disease scale

RCT: Randomized controlled trial

TE: Echo time

TMS: Transcranial magnetic stimulation

TR: Repetition time

\section{Declarations}

\section{Acknowledgements}

The authors are grateful to the staffs of the Chan Wei Wei Therapeutic Physical Mental Exercise Centre and to nurses and research assistants from the Department of Imaging and Interventional Radiology. We gratefully acknowledge study investigators and the dedication of study participants.

\section{Author's contributions}


LCW and SSM conceived and designed the study. LS and DW helped to conduct MRI scanning. STC and DPM helped to recruit the participants. HL developed this protocol, conducted rTMS and wrote this manuscript.

\section{Funding}

This work was supported by the General Research Fund of Research Grant Council of Hong Kong (GRF14111115).

\section{Availability of data and materials}

The dataset used and /or analyzed during the present study are available from the corresponding author on reasonable request.

\section{Ethics approval and consent to participate}

Ethics approval was obtained from the Joint Chinese University of Hong Kong-New Territories East Cluster Clinical Research Ethics Committee (The Joint CUHK-NTEC CREC) before the commencement of this study. Participants and their caregivers were acknowledged about the study before a decision for informed consent.

\section{Consent for publication}

All authors approved the final version of the manuscript for submission.

\section{Competing interests}

The authors have declared that no competing interests.

\section{Author details}

1. Department of Psychiatry, The Chinese University of Hong Kong, Hong Kong SAR, China. 2. Department of Medicine and Therapeutics, The Chinese University of Hong Kong, Hong Kong SAR, China. 3. Department of Imaging and Interventional Radiology, The Chinese University of Hong Kong, Hong Kong SAR, China

\section{References}

1. Jia J, Wei C, Chen S, Li F, Tang Y, Qin W, Zhou A. The cost of Alzheimer's disease in China and reestimation of costs worldwide. Alzheimers Dement. 2018;14:483-491.

2. Wise EA, Rosenberg PB, Lyketsos CG, eoutsakos JM. Time course of neuropsychiatric symptoms and cognitive diagnosis in National Alzheimer's Coordinating Centers volunteers. Alzheimers Dement. 2019;11:333-339. 
3. Ismail Z, Elbayoumi H, Fischer CE, Hogan DB, Millikin CP, Schweizer T, Fiest KM. Prevalence of depression in patients with mild cognitive impairment: a systematic review and meta-analysis. JAMA psychiatry. 2017;74:58-67.

4. Asmer MS, Kirkham J, Newton H, Ismail Z, Elbayoumi H, Leung RH, Seitz DP. Meta-Analysis of the Prevalence of Major Depressive Disorder Among Older Adults With Dementia. J Clin Psychiatry. 2018;79.

5. Fung AWT, Chan WC, Wong CSM, Chen EYH, Ng RMK, Lee EHM, Chiu HFK. Prevalence of anxiety disorders in community dwelling older adults in Hong Kong. Int Psychogeriatr. 2017;29:259-267.

6. Saczynski JS, Beiser A, Seshadri S, Auerbach S, Wolf PA, Au R. Depressive symptoms and risk of dementia: the Framingham Heart Study. Neurology. 2010;75:35-41.

7. Alexopoulos GS. Mechanisms and treatment of late-life depression. Transl Psychiatry. 2019;9:1-16.

8. Chase A. Altered functional connectivity in preclinical dementia. Nat Rev Neurol. 2014;10:609.

9. Öngür D, Drevets WC, Price JL. Glial reduction in the subgenual prefrontal cortex in mood disorders. PNAS. 1998;95:13290-13295.

10. Austin BP, Nair VA, Meier TB, Xu G, Rowley HA, Carlsson CM, Prabhakaran V. Effects of hypoperfusion in Alzheimer's disease. J Alzheimers Dis. 2011;26:123-133.

11. Xie C, Li W, Chen G, Ward BD, Franczak MB, Jones JL, Goveas JS. Late-life depression, mild cognitive impairment and hippocampal functional network architecture. Neuroimage Clin. 2013;3:311-320.

12. Zheng W, Cui B, Han Y, Song H, Li K, He Y, Wang Z. Disrupted regional cerebral blood flow, functional activity and connectivity in Alzheimer's disease: a combined ASL perfusion and resting state fMRI study. Front Neurosci. 2019;13,738.

13. Sayar GH, Ozten E, Tan O, Tarhan N. Transcranial magnetic stimulation for treating depression in elderly patients. Neuropsychiatr Dis Treat. 2013;9:501.

14. Koch G, Martorana A, Caltagirone $C$. Transcranial magnetic stimulation: emerging biomarkers and novel therapeutics in Alzheimer's disease. Neurosci Lett. 2020;719:134355.

15. Takeuchi N, Chuma T, Matsuo Y, Watanabe I, Ikoma K. Repetitive transcranial magnetic stimulation of contralesional primary motor cortex improves hand function after stroke. Stroke. 2005;36:26812686

16. George MS, Lisanby SH, Sackeim HA. Transcranial magnetic stimulation: applications in neuropsychiatry. Arch Gen Psychiatry. 1999;56:300-311.

17. Jorge RE, Moser DJ, Acion L, Robinson RG. Treatment of vascular depression using repetitive transcranial magnetic stimulation. Arch Gen Psychiatry. 2008;65:268-276.

18. Brys M, Fox MD, Agarwal S, Biagioni M, Dacpano G, Kumar P, Shukla AW. Multifocal repetitive TMS for motor and mood symptoms of Parkinson disease: a randomized trial. Neurology. 2016;87:19071915.

19. Shang Y, Wang $X$, Shang X, Zhang H, Liu Z, Yin T, Zhang T. Repetitive transcranial magnetic stimulation effectively facilitates spatial cognition and synaptic plasticity associated with increasing 
the levels of BDNF and synaptic proteins in Wistar rats. Neurobiol Learn Mem. 2016;134:369-378.

20. Cotelli M, Manenti R, Cappa SF, Zanetti O, Miniussi C. Transcranial magnetic stimulation improves naming in Alzheimer disease patients at different stages of cognitive decline. Eur J Neurol. 2008;15:1286-1292.

21. Baruch N, Burgess J, Pillai M, Allan CL. Treatment for depression comorbid with dementia. Evid Based Ment Health. 2019;22:167-171.

22. Kirsch I. The Placebo Effect in the Treatment of Depression and Anxiety. Front Psychiatry. 2019;10:407.

23. Rutherford BR, Wall MM, Brown PJ, Choo TH, Wager TD, Peterson BS, Roose SP. Patient expectancy as a mediator of placebo effects in antidepressant clinical trials. Am J Psychiatry. 2017;174:135-142.

24. American Psychiatric Association. Diagnostic and statistical manual of mental disorders (DSM-5®). American Psychiatric Pub. 2013.

25. Pieper MJ, Francke AL, van der Steen JT, Scherder, EJ, Twisk JW, Kovach CR, Achterberg WP. Effects of a stepwise multidisciplinary intervention for challenging behavior in advanced dementia: a cluster randomized controlled trial. J Am Geriatr Soc. 2016;64:261-269.

26. Fitzgerald PB, Hoy K, McQueen S, Maller JJ, Herring S, Segrave R, Daskalakis ZJ. A randomized trial of rTMS targeted with MRI based neuro-navigation in treatment-resistant depression.

Neuropsychopharmacology. 2009;34:1255-1262.

27. Fox MD, Buckner RL, White MP, Greicius MD, Pascual-Leone A. Efficacy of transcranial magnetic stimulation targets for depression is related to intrinsic functional connectivity with the subgenual cingulate. Biol Psychiatry. 2012;72:595-603.

28. Lu H, Chan SS, Lam LC. Localized Analysis of Normalized Distance from Scalp to Cortex and Personalized Evaluation (LANDSCAPE): Focusing on Age-and Dementia-Specific Changes. J Alzheimers Dis. 2019;67:1331-1341.

29. Smith JE, Peterchev AV. Electric field measurement of two commercial active/sham coils for transcranial magnetic stimulation. J Neural Eng. 2018;15:054001.

30. Schreiner AS, Hayakawa H, Morimoto T, Kakuma T. Screening for late life depression: cut-off scores for the Geriatric Depression Scale and the Cornell Scale for Depression in Dementia among Japanese subjects. Int J Geriatr Psychiatry. 2003;18:498-505.

31. Kramberger MG, Jelic V, Kåreholt I, Enache D, Jönhagen ME, Winblad B, Aarsland D. Cerebrospinal fluid Alzheimer markers in depressed elderly subjects with and without Alzheimer's disease. Dement Geriatr Cogn Disord Extra. 2012;2:48-56.

32. Wong A, Xiong YY, Kwan PW, Chan AY, Lam WW, Wang K, Mok VC. The validity, reliability and clinical utility of the Hong Kong Montreal Cognitive Assessment (HK-MoCA) in patients with cerebral small vessel disease. Dement Geriatr Cogn Disord. 2009;28:81-87.

33. Lu H, Chan SS, Fung AW, Lam LC. Utility of Montreal Cognitive Assessment (Hong Kong Version) in the Diagnosis of Mild Neurocognitive Disorders (NCD): NCD due to Alzheimer Disease (NCD-AD) and NCD due to Vascular Disease (NCD-Vascular). J Am Med Dir Assoc. 2016;17:366-367. 
34. Wong A, Cheng ST, Lo ES, Kwan PW, Law LS, Chan AY, Mok V. Validity and reliability of the neuropsychiatric inventory questionnaire version in patients with stroke or transient ischemic attack having cognitive impairment. Int J Geriatr Psychiatry. 2014;27:247-252.

35. Logsdon RG, Gibbons LE, McCurry SM, Teri L. Quality of life in Alzheimer's disease: patient and caregiver reports. J Ment Healt Aging. 1999;5:21-32.

36. Brunoni AR, Lopes M, Fregni F. A systematic review and meta-analysis of clinical studies on major depression and BDNF levels: implications for the role of neuroplasticity in depression. Int J Neuropsychopharmacol. 2008;11:1169-1180.

37. Lu H, Lam LCW. Cathodal skin lesions induced by transcranial direct current stimulation (tDCS). Neuromodulation. 2019;22:989-991.

38. Monroe TB, Gibson SJ, Bruehl SP, Gore JC, Dietrich MS, Newhouse P, Cowan RL. Contact heat sensitivity and reports of unpleasantness in communicative people with mild to moderate cognitive impairment in Alzheimer's disease: A cross-sectional study. BMC Med. 2016;14:74.

39. Pascual-Leone A, Rubio B, Pallardó F, Catalá MD. Rapid-rate transcranial magnetic stimulation of left dorsolateral prefrontal cortex in drug-resistant depression. Lancet. 1996;348:233-237.

40. George MS, Nahas Z, Molloy M, Speer AM, Oliver NC, Li XB, Ballenger JC. A controlled trial of daily left prefrontal cortex TMS for treating depression. Biol Psychiatry. 2000;48:962-970.

41. Schutter DJLG. Antidepressant efficacy of high-frequency transcranial magnetic stimulation over the left dorsolateral prefrontal cortex in double-blind sham-controlled designs: a meta-analysis. Psychol Med. 2009;39:65-75.

42. Gu SY, Chang MC. The effects of $10-\mathrm{Hz}$ repetitive transcranial magnetic stimulation on depression in chronic stroke patients. Brain Stimul. 2017;10:270-274.

43. Figiel GS, Epstein C, McDonald WM, Amazon-Leece J, Figiel L, Saldivia A, Glover S. The use of rapidrate transcranial magnetic stimulation (rTMS) in refractory depressed patients. J Neuropsychiatry Clin Neurosci. 1998;10:20-25.

44. Fabre I, Galinowski A, Oppenheim C, Gallarda T, Meder JF, De Montigny C, Poirier MF. Antidepressant efficacy and cognitive effects of repetitive transcranial magnetic stimulation in vascular depression: an open trial. Int J Geriatr Psychiatry. 2004;19:833-842.

45. Mosimann UP, Schmitt W, Greenberg BD, Kosel M, Müri RM, Berkhoff M, Schlaepfer TE. Repetitive transcranial magnetic stimulation: a putative add-on treatment for major depression in elderly patients. Psychiatry Res. 2004;126:123-133.

46. Manes F, Jorge R, Morcuende M, Yamada T, Paradiso S, Robinson RG. A controlled study of repetitive transcranial magnetic stimulation as a treatment of depression in the elderly. Int Psychogeriatr. 2001;13:225-231.

47. Taylor SF, Ho SS, Abagis T, Angstadt M, Maixner DF, Welsh RC, Hernandez-Garcia L. Changes in brain connectivity during a sham-controlled, transcranial magnetic stimulation trial for depression. J Affect Disord. 2018;232:143-151. 
48. Berlim MT, Van den Eynde F, Tovar-Perdomo S, Daskalakis ZJ. Response, remission and drop-out rates following high-frequency repetitive transcranial magnetic stimulation (rTMS) for treating major depression: a systematic review and meta-analysis of randomized, double-blind and sham-controlled trials. Psychol Med. 2014;44:225-239.

49. Gross M, Nakamura L, Pascual-Leone A, Fregni F. Has repetitive transcranial magnetic stimulation (rTMS) treatment for depression improved? A systematic review and meta-analysis comparing the recent vs. the earlier rTMS studies. Acta Psychiatr Scand. 2007;116:165-173.

50. Conelea CA, Philip NS, Yip AG, Barnes JL, Niedzwiecki MJ, Greenberg BD, Carpenter LL. Transcranial magnetic stimulation for treatment-resistant depression: Naturalistic treatment outcomes for younger versus older patients. J Affect Disord. 2017;217:42-47.

51. Iriarte IG, George MS. Transcranial magnetic stimulation (TMS) in the elderly. Curr Psychiatry Rep. 2018;20:6.

52. Cuijpers P, Karyotaki E, Eckshtain D, Ng MY, Corteselli KA, Noma H, Weisz JR. Psychotherapy for Depression Across Different Age Groups: A Systematic Review and Meta-analysis. JAMA psychiatry. 2020;77:694-702.

53. Jodoin VD, Miron JP, Lespérance P. Safety and efficacy of accelerated repetitive transcranial magnetic stimulation protocol in elderly depressed unipolar and bipolar patients. Am J Geriatr Psychiatry. 2019;27:548-558.

\section{Tables}


Table 1

Baseline demographics and clinical characteristics of all participants

\begin{tabular}{|c|c|c|c|c|}
\hline & $\begin{array}{l}\text { Active rTMS } \\
(n=27)\end{array}$ & $\begin{array}{l}\text { Sham rTMS } \\
(n=28)\end{array}$ & $\begin{array}{l}t \text { value } \\
(x 2)\end{array}$ & $P$ value \\
\hline Age & $69.2 \pm 7.1$ & $73.7 \pm 7.2$ & -2.39 & 0.02 \\
\hline $\operatorname{Sex}(F / M)$ & $19 / 8$ & $19 / 9$ & 0.04 & 0.84 \\
\hline Education (years) & $7.3 \pm 4.4$ & $7.5 \pm 5.2$ & -0.10 & 0.92 \\
\hline CIRS & $4.9 \pm 3.0$ & $4.1 \pm 1.9$ & 1.17 & 0.25 \\
\hline CSDD & $12.7 \pm 4.3$ & $11.5 \pm 3.7$ & 1.11 & 0.27 \\
\hline HK MoCA & $20.3 \pm 5.3$ & $19.9 \pm 6.1$ & 0.29 & 0.78 \\
\hline QoL-AD & $29.4 \pm 5.8$ & $27.1 \pm 7.4$ & 1.06 & 0.29 \\
\hline CNPI & $11.1 \pm 8.7$ & $10.6 \pm 9.4$ & 0.18 & 0.86 \\
\hline \multicolumn{5}{|c|}{$\begin{array}{l}\text { Note. Data are raw scores and presented as mean } \pm \text { SD. Abbreviations: CIRS = Cumulative Illness } \\
\text { Rating Scale; CSDD = Cornell Scale for Depression in Dementia; HK MoCA = Montreal Cognitive } \\
\text { Assessment Hong Kong version; QoL-AD = Quality of Life-AD; CNPI = Chinese Neuropsychiatric } \\
\text { Inventory. }\end{array}$} \\
\hline
\end{tabular}

Table 2

Score changes of primary outcomes across the intervention groups

\begin{tabular}{|c|c|c|c|c|}
\hline & $\begin{array}{l}\text { Active rTMS } \\
(\mathrm{n}=25)\end{array}$ & $\begin{array}{l}\text { Sham rTMS } \\
(n=27)\end{array}$ & t value & $P$ value \\
\hline \multicolumn{5}{|c|}{ Post-intervention (T1) } \\
\hline CSDD & $3.24 \pm 3.53$ & $2.15 \pm 3.22$ & 1.17 & 0.25 \\
\hline HK MoCA & $2.20 \pm 1.94$ & $1.89 \pm 2.89$ & 0.46 & 0.65 \\
\hline \multicolumn{5}{|c|}{ 6th week follow-up (T2) } \\
\hline CSDD & $2.84 \pm 2.93$ & $2.22 \pm 2.94$ & 0.76 & 0.45 \\
\hline HK MoCA & $2.20 \pm 2.47$ & $2.11 \pm 2.83$ & 0.12 & 0.90 \\
\hline \multicolumn{5}{|c|}{ 12th week follow-up (T3) } \\
\hline CSDD & $3.24 \pm 2.83$ & $2.56 \pm 4.84$ & 0.62 & 0.54 \\
\hline HK MoCA & $3.04 \pm 2.42$ & $1.26 \pm 3.37$ & 2.17 & 0.03 \\
\hline
\end{tabular}


Table 3

Raw scores of primary outcomes across the intervention groups

\begin{tabular}{|lllll|}
\hline & $\begin{array}{l}\text { Active rTMS } \\
(\mathbf{n}=\mathbf{2 5})\end{array}$ & $\begin{array}{l}\text { Sham rTMS } \\
(\mathbf{n}=\mathbf{2 7})\end{array}$ & t value & P value \\
\hline Post-intervention (T1) & & & \\
\hline CSDD & $9.28 \pm 4.67$ & $9.22 \pm 3.69$ & 0.05 & 0.96 \\
\hline HK MoCA & $22.08 \pm 5.15$ & $21.33 \pm 6.49$ & 0.46 & 0.65 \\
\hline 6th week follow-up (T2) & & & 0.46 & 0.65 \\
\hline CSDD & $9.68 \pm 4.60$ & $9.15 \pm 3.80$ & 0.29 & \\
\hline HK MoCA & $22.08 \pm 5.73$ & $21.56 \pm 7.04$ & 0.77 \\
\hline 12th week follow-up (T3) & & & 0.36 & 0.24 \\
\hline CSDD & $9.28 \pm 4.18$ & $8.81 \pm 4.96$ & 1.19 & \\
\hline HK MoCA & $22.92 \pm 5.25$ & $20.70 \pm 7.82$ & \\
\hline $\begin{array}{l}\text { Note. Data are raw scores and presented as mean } \pm \text { SD. Abbreviations: CSDD = Cornell Scale for } \\
\text { Depression in Dementia; HK MoCA = The Montreal Cognitive Assessment Hong Kong version. }\end{array}$ \\
\hline
\end{tabular}

Figures 


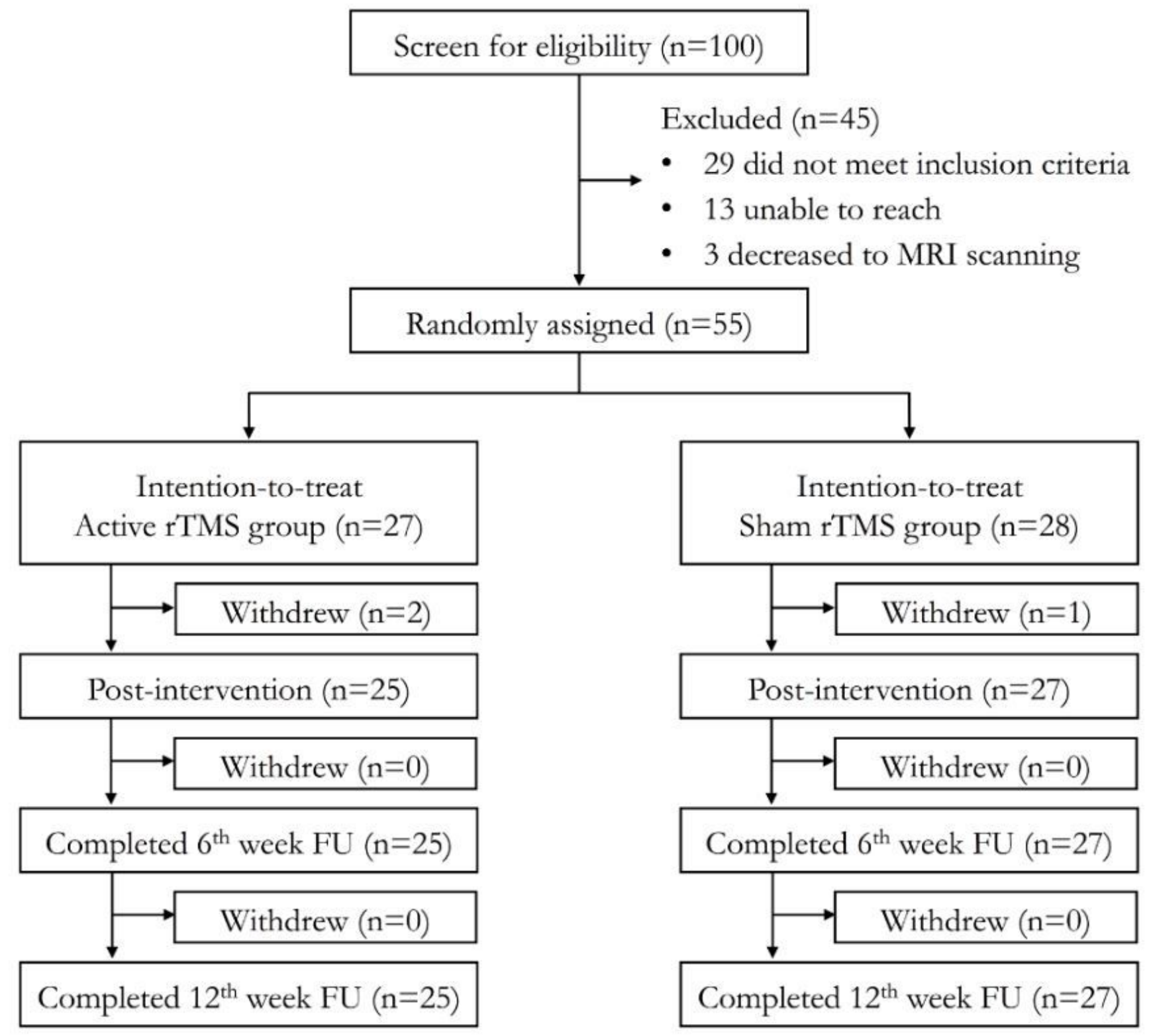

Figure 1

Consolidated Standards of Reporting Trials (CONSORT) flow diagram for neuronavigated HF-rTMS clinical trial. 
(A)

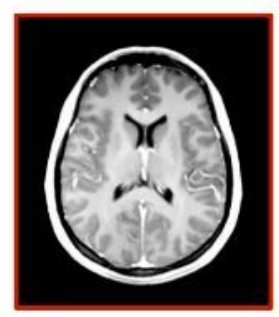

Pre-treatment sMRI scans

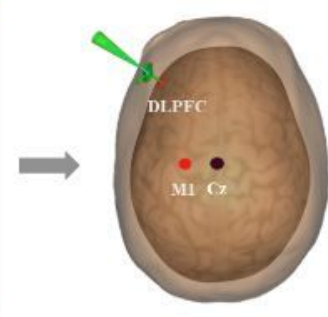

Localizing Targets
(B)

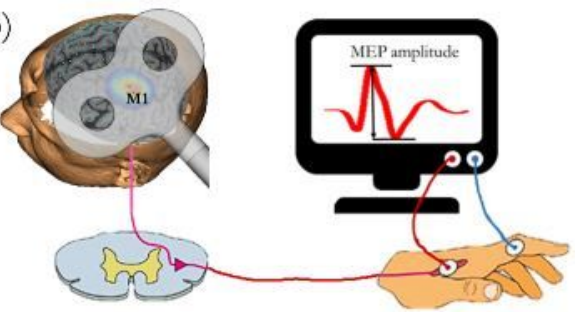

MT

Measurement
(C)

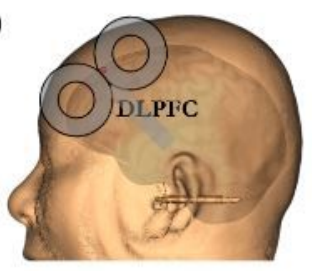

Stimulating

Left DLPFC

\section{Figure 2}

Pipeline of HF-rTMS treatment, including (a) Individual MRI scanning, (b) reconstructing and localizing targets, (c) measuring the motor evoked potentials (MEP), (d) daily rTMS treatment.

(A)

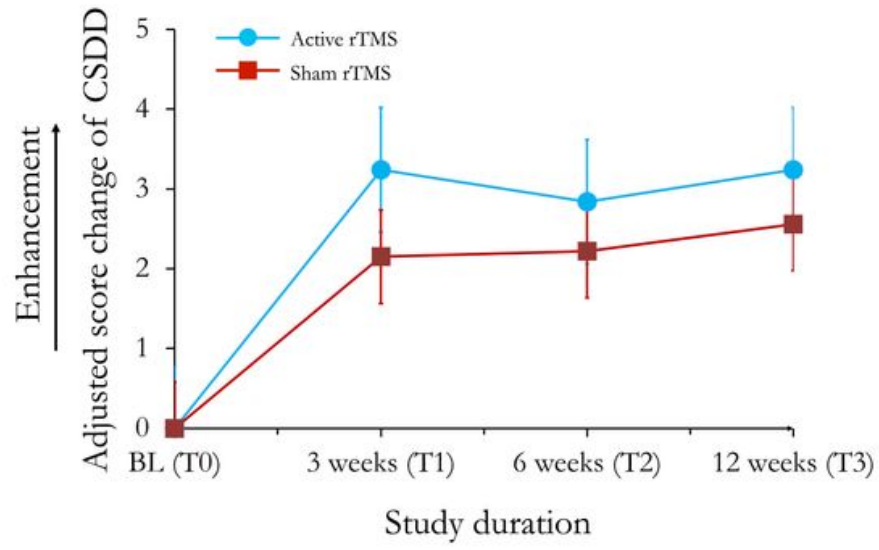

(B)

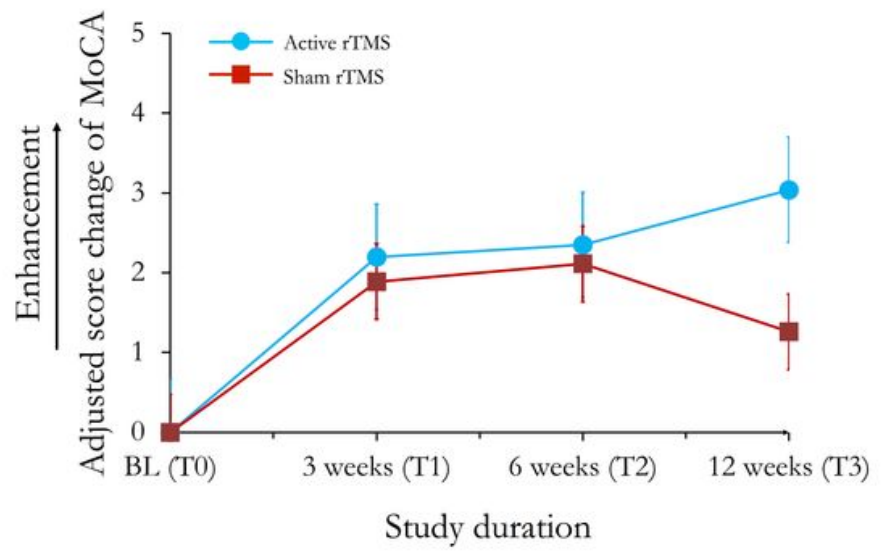

Figure 3

Primary outcome measures of the Cornell Scale for Depression in Dementia (CSDD) and the Montreal Cognitive Assessment Hong Kong version (HK MoCA). Panel A shows the results for the score change from baseline in the score on CSDD (Higher score indicating great enhancement). Error bars represent the standard error (SEM). 

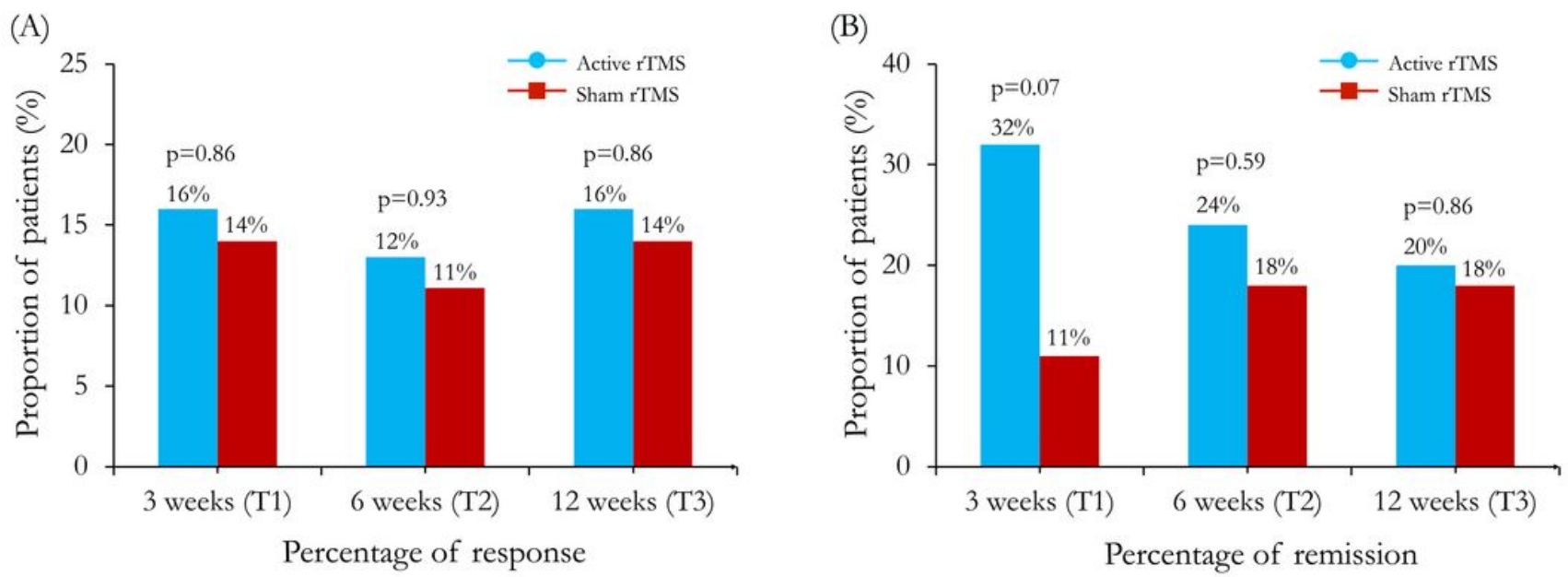

\section{Figure 4}

Differences of response rate and remission rate in active rTMS and sham rTMS groups across all time points. 